\title{
An Investigation of the Prevalence of Upper Limb Neuropathies in Different Types of College Musicians by Use of Neurometrix Device
}

\author{
Saunders Jones Jr MD (Corresponding author) \\ Dept of Biology and Physics, Kennesaw State University \\ 1000 Chastain Road, \#1202, Science and Mathematics \\ Bldg \#12, Rm SC337, Kennesaw, Georgia \\ Tel: 770-423-6498 E-mail: Sjones12@kennesaw.edu \\ Christi Hernandez \\ Dept of Biology, Kennesaw State University \\ 1000 Chastain Road, \#1202, Science and Mathematics \\ Bldg \#12, Rm SC337, Kennesaw, Georgia
}

\begin{abstract}
In general, people who perform repetitive motions are often vulnerable to repetitive strain injuries. Because musicians must execute the same motion over and over again while practicing and performing their music, they are an example of a group that often develops these repetitive strain injuries. More specifically, musicians are known for developing neuropathies in their upper limbs, with carpal tunnel syndrome and cubital tunnel syndrome being most common. However, because of varying playing postures, all musicians may not be at equal risk for developing these two neuropathies, so the purpose of this study was to identify which musician group has the highest risk of developing median and ulnar neuropathies. Results of this study show that the prevalence of carpal tunnel syndrome and cubital tunnel syndrome is fairly low, and that tingling is one of the first signs of these diseases. Also, brass players may have the highest risk of developing carpal tunnel syndrome, as only this musician group had a significant p-value when its incidence rate of carpal tunnel syndrome was compared to the incidence rate found in the general population. Finally, the results suggest that there may be a negative correlation between performing a warm-up routine and experiencing tingling. Therefore, music schools should teach all students, and especially brass players, to be aware of tingling and to take appropriate preventative measure such as warming-up in order to keep the incidence rates of carpal tunnel syndrome and cubital tunnel syndrome as low as possible.
\end{abstract}

Keywords: Carpal tunnel, Musicians, Occupational exposure, Nerve conduction studies

\section{Introduction: Clinical parameters of Carpal Tunnel Syndrome and Cubital Tunnel Syndrome}

\subsection{Etiologies of Carpal Tunnel Syndrome}

In many instances in which a person is required to perform the same physical action over and over again, a certain amount of stress may be put onto the overused body parts, which can result in a repetitive strain injury/cumulative trauma. These injuries can affect anyone who performs these repetitive types of motions, but are especially prevalent in typists, carpenters, electronics assembly line workers, textile workers, butchers, grocery checkers, packers, and musicians, as these groups of people perform repetitive and sometimes forceful motions almost every day (Cummings et al. 1989). In particular, a number of musicians tend to develop upper limb neuropathies as a common type of repetitive strain injury, with carpal tunnel syndrome and cubital tunnel syndrome being two of the most common types of neuropathies.

\subsection{Physiologic Changes of Carpal Tunnel Syndrome}

Carpal tunnel syndrome is caused by an abnormal amount of pressure being placed on the median nerve as it passes through the carpal canal of the wrist (the canal walls are composed of the carpal bones and the transverse carpal 
ligament, which enclose the median nerve and nine flexor tendons). This can either occur through "factors that increase the volume of the contents of the carpal canal" (intrinsic carpal tunnel syndrome), or through factors that decrease the actual size of the canal (extrinsic carpal tunnel syndrome) (Kerwin 1996). If this excessive amount of pressure occurs regularly, it can cause damage to the median nerve by removing part of the myelin sheath surrounding the nerve, which then slows the conduction velocities of both motor and sensory electrical impulses that are being sent through that nerve.

\subsection{Presenting Symptoms}

The first symptoms of carpal tunnel syndrome include pain, weakness, or paresthesias in the first three digits and in the radial half of the ring finger, and the symptoms often increase in severity at night as many subjects tend to sleep with their wrists in a flexed or extended position, which increases the pressure inside the carpal canal (Strickland 2001). If the condition is then left untreated, demyelination of the nerve can continue and can severely limit use of the hand, as the damaged median nerve will not dependably conduct an electrical impulse to the neuromuscular junction, resulting in limited muscular contraction and eventual atrophy of the innervated muscles.

\subsection{Diagnosis}

Carpal tunnel syndrome can be diagnosed in a couple of ways, the first of which is provocative tests including Tinel's test and Phalen's test. Though these types of tests are somewhat useful, they should not be the only diagnostic technique used, as Gerr and Letz (1998) found that sensitivity for the Tinel's test can range from $26 \%$ to $79 \%$, and that sensitivity for the Phalen's sign can range from $10 \%$ to $88 \%$. Therefore, the best way to diagnose carpal tunnel syndrome is through nerve conduction studies. During these studies, the medical professional is often most interested in distal motor latency, which is the amount of time it takes for the nerve impulse to travel from the site of the stimulus (such as some specified point along the median nerve) to the innervated muscle (such as the thenar muscles). A person who is affected with carpal tunnel syndrome will usually exhibit a longer than normal distal motor latency due to the damaged myelin sheath of the median nerve.

\subsection{Presenting symptoms and Physiology of Cubital Tunnel Syndrome}

The second common upper limb neuropathy found in musicians is cubital tunnel syndrome, which involves entrapment of the ulnar nerve at the elbow. Similar to carpal tunnel syndrome, cubital tunnel syndrome is caused by excessive pressure on the ulnar nerve as it passes through the cubital tunnel on the outside edge of the elbow. This excessive pressure can damage the myelin sheath and slow conduction velocities, which can prevent appropriate contraction of the innervated muscles.

\subsection{Physiology of Cubital Tunnel Syndrome}

Also similar to carpal tunnel syndrome are the primary symptoms of cubital tunnel syndrome. The initial symptoms of cubital tunnel syndrome include pain, weakness, and paresthesias in the little finger and ring finger, as these are the digits that are innervated by the ulnar nerve. If left untreated, the myelin sheath will likely suffer an increasing amount of damage, which can again reduce activity at the neuromuscular junction and cause atrophy of the innervated muscles as well as "clawing of the fourth and fifth digits" (Robertson 2005)

\subsection{Diagnosis of Cubital Tunnel Syndrome}

Cubital tunnel syndrome is usually diagnosed via nerve conduction studies involving F-waves. To do this, a specific section of the ulnar nerve is electrically stimulated, which causes electrical impulses to travel both orthodromically and antidromically. The orthodromic impulse will of course reach the innervated muscle first, and the muscle contraction that results creates the $\mathrm{M}$-wave. At the same time, the antidromic impulse first travels to the spinal cord and will then reverse directions and eventually reach the neuromuscular junction, where it will cause another muscular contraction that will produce the F-wave. Because the impulse that produces the F-wave must travel through the cubital tunnel during its journey to and from the spinal cord, the latency, amplitude, and duration of the resulting F-wave can be examined for abnormalities that are characteristic of cubital tunnel syndrome.

\section{Risk Exposure of different Musicians to these Syndromes}

Now knowing the causes and diagnostic techniques of these two neuropathies, it is possible to examine the playing postures of four different types of musicians in order to see which musicians may be especially at risk for one or both of these disorders.

\subsection{Brass Players}

Brass players are one type of musician, and include people who play the trumpet, tuba, trombone, and French horn. In order to play these instruments, the musician does not usually need to have an especially strong grip on the instrument, nor is this gripping hand in an awkward position. The other hand, known as the valve hand, may deviate slightly from 
neutral position, but overall is still fairly free to move. This neutral positioning probably means that brass players would not be at a high risk of developing either of these two neuropathies.

\subsection{Woodwind Players}

A second group of musicians are the people who play woodwind instruments such as the clarinet, saxophone, and flute. Musicians who play the flute are required to engage in extension and ulnar deviation of the left hand, which could put them at a higher risk for carpal tunnel syndrome. Additionally, playing the saxophone and clarinet often requires wrist flexion and high levels of thumb stress, which could also lead to carpal tunnel syndrome.

\subsection{Percussionists}

A third group of musicians are percussionists, such as those people who play piano or drums. The instruments are often played with a great deal of force and often require flexion of the wrist when playing the instrument, which could put excess pressure on the median nerve and increases this group's risk of developing carpal tunnel syndrome. In fact, Gohl (2006) found that sixteen percent of university pianists exhibited evidence of carpal tunnel syndrome, which is indeed higher than the two to three percent incidence rate that is found in the general population (Atroshi 1999).

\subsection{String Instruments}

A fourth group of musicians are those people who play string instruments such as violin, viola, cello, and bass. These musicians are often required to hold the instrument quite firmly with a flexed wrist, which could put them at higher risk for carpal tunnel syndrome. In addition, violins and violas are also held in place by the chin, which forces the neck into a flexed position, and can therefore cause damage to the median nerve as it branches off from the brachial plexus. Also, because violinists and violists must constantly flex the left elbow to support their instrument, this arm could be at a higher risk of developing cubital tunnel syndrome. In a study of young adult violinists, Bowie et al. (2000) found that thirty-five percent of the subjects showed early electrophysiologic signs of carpal tunnel syndrome, which is again higher than the incidence rate of the general population.

\subsection{Hypothesis related to groups}

Therefore, knowing the playing requirements of each type of musician, it is hypothesized that the type of instrument played (brass, woodwind, percussion, or string) may be a determining factor in the amount of nerve damage present on the median and ulnar nerves of Kennesaw State University music students. In addition, it is predicted that because the string group usually has the most strained playing postures, this group will be most likely to have nerve damage, while the group with the least strained playing posture (the brass group) will be least likely to show signs of median or ulnar nerve damage.

In order to test this hypothesis, Kennesaw State University music education or music performance majors were randomly selected to participate in the study. They were asked to fill out a survey that will give the researchers background information about each subject's medical history, health habits, and musical habits. After this, the health of both the median and ulnar nerve was assessed for each subject using the NeuroMetrix NC-Stat electrodiagnostic system, and the resulting data for each group was analyzed.

\section{Methods}

\subsection{Musician Type Findings}

For this experiment, the independent variable was the musician type. It was applied by asking each subject to state which instrument is his or her most major instrument. Based on the answer to this question, the subject was placed into one of four major groups: brass, woodwind, percussion, or string. The dependent variable was the health of both the median and ulnar nerves. This was measured using the NC-Stat system, using distal motor latency as one of the most important pieces of data describing the health of the median nerve and F-wave latency as one of the most important pieces of data describing the health of the ulnar nerve. In addition, the number of median and ulnar nerve "flags" was examined, with a flag being defined as any result deemed by the NeuroMetrix system to be abnormal (these abnormalities can range from being borderline abnormal to severely abnormal).

\subsection{Age Contribution to Findings}

In addition, a number of variables were also controlled during this experiment, the first of which was the age of the subjects. Because all of the subjects were undergraduate students in college, they were all around the same age. This is important because neuropathies generally tend to increase in severity in older people, so if the subjects were not all around the same age, one musician group could show less healthy nerves simply because it had a larger number of older individuals.

\subsection{Measuring Device standardization}

The same NC-Stat system was used to measure each subject. This ensured that differences in machine sensitivity did not bias the information. In addition, because the NC-Stat system corrects for the temperature of the subject, it ensured 
that some subjects did not have longer latency periods just because their body temperature was colder than other subjects' body temperatures. Finally, after the data was collected from each subject, it was compared to a large database of national averages, so this database (provided by NeuroMetrix) acted as the control population.

\subsection{Data Gathering and Measurment of Subjects}

In order to carry out the actual experiment, the test subjects were selected from the group of music education and music performance majors at Kennesaw State University. To do this, a researcher went to the music building, discussed the study with available music students, and obtained email addresses for those students who were interested in participating. An email was then sent to the interested students describing the study in more detail and informing the students about how they could make an appointment time.

\subsubsection{Survey}

When the subject arrived for his appointment, he was given a booklet that included an informed consent as well as a survey. The first portion of the survey inquired about physical and mental health history, while the second part of the survey asked the participant to answer questions regarding his or her major musical instrument, musical history, daily/weekly musical routines, and music related medical problems. It is important to note that if the participant asked for clarification, "tingling" was defined as a sensation of pins and needles or numbness in the fingers and "warm-up" was defined as any stretching or other motions that were done prior to playing one's instrument.

\subsubsection{Nerve Measurements}

After the survey was completed, the forearm of the student was cleaned and the electrodes of the right median nerve biosensor were applied. This biosensor was then connected to the NC-Stat system, the subject information was entered, and then the test was started. During the test, the NC-Stat system sends electric impulses of gradually increasing strength through the appropriate nerve, which makes the innervated muscles contract. This muscular contraction is recorded by sensory electrodes, the data is stored in the machine, and is later compared against the control database discussed earlier. The same process was then repeated for the left median nerve, the right ulnar nerve, and finally the left ulnar nerve.

\subsection{Data Analysis and Statistics}

Then, after the data was collected, each subject was placed into one of four musician groups: brass, woodwind, percussion, or strings. General descriptive statistics such as the proportion of results that were outside the reference range and the distribution of results in terms of percentile groups were then performed. More detailed descriptive statistics were also performed in order to compare reported tingling, electrophysiological slowing, and reported warm-up in each of the musician groups. Chi-square tests were also performed in order to evaluate the incidence rate of carpal tunnel syndrome in each of the musician groups versus the incidence rate in the general population.

Finally, in order for the obtained data to support the study's hypothesis that the type of instrument played is a determining factor on the amount of median or ulnar nerve damage seen in college musicians, at least one of the chi-square tests comparing the incidence of carpal tunnel in the general population to the incidence rate in a certain musician population would have to produce a statistically significant p-value (less than 0.05). In addition, in order for the prediction that string players are at the highest risk for nerve damage to be supported, this group would have to show the most electrophysiological evidence of slowing (for instance, having the greatest proportion of members who were outside the reference range) or other abnormalities (having the greatest average number of median and ulnar flags). Finally for the data to support the second prediction that brass players are at the lowest risk for nerve damage, this group would have to show the least electrophysiological evidence of slowing or other abnormalities.

\section{Results}

\subsection{Relationship between this group and the general population}

Before looking at the differences between musician groups, it was first important to note the general trends that were found in this population of musicians. First, the overall incidence rate of carpal tunnel syndrome in the musician population was not significantly different from the incidence rate in the general population, as a chi-square test comparing these rates returned a p-value of 0.2092. Additionally, figure 1 shows that only a small proportion of musicians had values outside of the reference range, which again was the criteria used to diagnose someone as having electrical evidence of nerve compression and carpal tunnel syndrome or cubital tunnel syndrome.

In addition, figure 2 shows the gradation of the test results, and again highlights the fact that most musicians do not have abnormal conduction rates, and that a large proportion of musicians are at or above the fiftieth percentile when compared to the average population. When examining this figure, it is important to remember that the only people who could be neuroelectrically diagnosed as having carpal tunnel syndrome or cubital tunnel syndrome are subjects found in the lowest percentile group. 


\subsection{Findings of Measured Nerve Latency vs Symptomatic Subjects}

A second general trend shows that a large proportion of musicians who experience tingling in their fingers do not have any significant electrophysiological signs of carpal tunnel syndrome. In figure 3, over sixty percent of musicians reported tingling, but of that sixty percent, only $16.7 \%$ had a right DML below the twentieth percentile, and only $16.7 \%$ had a left DML below the twentieth percentile.

Also, for all musicians who reported tingling, the average percentile for DML-R and for DML-L was greater than 50\%, therefore showing that the average musician who reports tingling does not have any evidence of objective changes in his nerve studies.

\subsection{Measuring Reported symptoms vs Instrument Played}

In addition, a number of results were calculated regarding different categories of musicians. Figure 4 shows that symptomatically, percussion players had the most reported subjective symptoms (as all percussion players reported tingling), while string players had the least reported subjective symptoms (with only twenty percent reporting tingling).

\subsection{Measurement of DML in each studied instrument group}

In addition to looking at different symptoms between groups, the researchers also looked at electrophysiological differences between groups. Table 1 highlights these differences, and shows that brass musicians had the most problems, while all of the other groups had no electrophysiological confirmations of carpal tunnel syndrome or cubital tunnel syndrome.

\subsection{Comparing Study group prevalence vs general population.}

Chi-square tests were also used to compare the prevalence of carpal tunnel syndrome in the general population to the prevalence in each of the musician groups, and as shown in table 2, only the brass group showed a statistically significantly higher rate than that found in the general population.

\subsection{Severity of the Measured Nerve changes in the Brass Player Group}

Also, as figure 5 and figure 6 show, brass players had the highest average number of median and ulnar nerve flags (flags were determined by the NC-Stat system and represented mild to severe abnormalities in any of the electrical values).

\subsection{Affect of Warm Up on presence of Symptomatology in all groups}

Finally, the researchers examined the correlation between performing a warm-up and the prevalence of symptoms. As figure 7 shows, musicians who perform a warm-up routine prior to playing are less likely to report experiences of tingling than those who do not warm-up prior to playing.

Figure 8 also shows a similar negative correlation between warming-up and reports of tingling, especially for the brass and string groups.

\section{Discussion}

\subsection{Trends in Data}

\subsubsection{Low incidence of Disease}

By examining the above data, a number of general trends can be identified. First, it seems that there is not a large prevalence of carpal tunnel syndrome or cubital tunnel syndrome in this population of college musicians. Figure 1 shows that only a small proportion of students had values outside of the reference range for DML-R, DML-L, F-wave R or F-wave left, and all of the values that were outside the reference range are from three students who each had evidence of slowing in more than one nerve. Figure 2 also shows a low prevalence of electrical signs of carpal tunnel syndrome or cubital tunnel syndrome, as most musicians were not even below the tenth percentile and a large number of musicians were at or above the fiftieth percentile.

\subsubsection{First Sign of Carpal Tunnel is usually tingling}

Secondly, it seems that there is no earlier sign or symptom of carpal tunnel syndrome than a subject's reports of tingling. Figure 3 shows that most musicians who report tingling do not have any electrical slowing in the median nerve. Because sensations of tingling appear to be more sensitive than electrophysiological evidence, musicians should generally have the ability to identify the start of a problem without expensive nerve testing.

\subsubsection{Subjects did not demonstrate Failure to report symptoms}

Also, it also seems that there is no failure to report symptoms. If students who had signs of electrical slowing had failed to report symptoms, this may have shown the effect of a professional pressure to play without complaints in order to get more jobs or better jobs. However, all students that had values outside of the reference range also reported having tingling in their fingers, so it appears that students are not afraid to report symptoms. 


\subsubsection{Further Analysis}

Brass Players had more abnormal studies which does not substatiate "unnatural position theory" as etiology of Carpal tunnel Syndrome or Cubital Tunnel Syndrome in this population

In addition to these general conclusions, a number of more specific conclusions can be made regarding this experiment's original hypothesis. This experiment tested the hypothesis that the type of instrument played may be a determining factor in the amount of nerve damage present in the median and ulnar nerves of Kennesaw State University musicians. In order for this hypothesis to be supported, one category of musician would have to show a significantly greater amount of electrical slowing than other categories of musicians. As table 1 shows, only brass players had values outside of the reference range for any of the four electrical values (DML-R, DML-L, F-wave right, and F-wave left). In addition, only brass players had a statistically significant $p$-value when the incidence rate of carpal tunnel syndrome in that category of musicians was compared to the average incidence rate of $2.5 \%$ in the general population. Figure 5 also shows that brass players had the highest average number of flags, again indicating more electrical evidence of nerve damage. Therefore, because brass players showed more electrical evidence of nerve damage than any other musician category, the researchers fail to falsify the hypothesis that the type of instrument played may be a determining factor in the amount of nerve damage present.

It was originally predicted that because string players generally have the most strained playing posture, this group would have the most nerve damage, and because brass players have the least strained playing posture, this group would have the least nerve damage. However, as stated above, the brass group was the only group to show a statistically significantly greater prevalence of carpal tunnel syndrome when compared to the general population. Also, $62.5 \%$ of brass players had experienced tingling while only $20 \%$ of string players had experienced tingling.

\subsubsection{Causes of Discrepancies in Hypothesis}

These results are basically opposite of what the researchers had originally predicted, but a closer look into personal care habits may explain this unanticipated trend. First of all, $100 \%$ of the string players reported that they perform a warm-up routine prior to playing their instrument, but only $12.5 \%$ of brass players reported performing such a warm-up routine. This may mean that there is some sort of negative correlation between performing a warm-up routine and experiencing tingling. In addition, all string players reported exercising more than two times per week, while only $16.7 \%$ of brass players reported exercising more than two times per week. This suggests that there may also be some sort of negative correlation between exercising and experiencing tingling.

\section{Conclusions and Reccomendations}

When analyzing the overall experimental design of this study, it seems that the experiment did adequately test the hypothesis that the type of instrument played may be a determining factor in the amount of nerve damage present in the median and ulnar nerves. The independent variable was of course the type of instrument played (brass, percussion, woodwind, or string), and was determined by the researchers after analyzing each participant's response to the survey question "Instrument that you now think is your major instrument?" There were a number of dependent variables. Two of the most important dependent variables were the distal motor latency of the median nerves and the F-wave latency of the ulnar nerves. These were measured by the NC-Stat system, so they were fairly trouble-free except for the fact that a few ulnar sensors did not work properly, so a few of the F-wave latencies could not be computed. Other dependent variables included reports of tingling, warm-up, and weekly exercise, and the respondents simply circled the appropriate multiple choice answer, so this was also fairly trouble-free (some subjects did not see the multiple choice answers, but could still be put into an appropriate category fairly easily). The experimental design also included a number of control variables. The body temperature of the subject, gender, height, and weight was taken into account by the NC-Stat system as it was calculating the electrical values, so these variables were adequately controlled. Also, almost all subjects were either in their late teens or early twenties and the NC-Stat system also helped to adjust for age, so this was fairly well controlled. However, if may be important to note that one of the brass players who had electrophysiological signs of both carpal tunnel syndrome and cubital tunnel syndrome was thirty-seven years old, which was in fact older than most of the other subjects.

Also, when looking at similar studies, these results appear to correspond nicely with other findings. For example, Logue (2005) found that in a study of fourteen university cellists, "all subjects were found to have normal nerve conduction studies of the median and ulnar nerves in both extremities when compared with a chart of normal values." Therefore, Logue's findings are very similar to this study's findings, as both showed no abnormal conduction in string players. Also, Gohl (2006) had similar findings in a study of nineteen university pianists, as she found that all subjects had normal conduction values when compared to a chart of normal values. However, when she compared median and ulnar latencies in the same and opposite hand, she found that three of the nineteen pianists $(16 \%)$ showed early evidence of median neuropathy. Finally, Bowie (2000) also had similar findings in a study of twenty young adult violinists. Bowie found that all subjects had normal median and ulnar conduction values when compared to a chart of normal values. 
However, like Gohl, Bowie found that when the median nerve conduction was compared to ulnar nerve conduction in the same extremity, seven of the twenty violinists (35\%) showed evidence of early median neuropathy.

Therefore, the results of our study tend to agree with previous research. In addition, because these results suggested negative correlations between performing a warm-up routine and experiencing tingling and between exercising more than two times per week and tingling, it may be interesting to do further study in order to see if these correlations are indeed significant In conclusion, it seems that tingling is usually the first symptom of early carpal tunnel syndrome and cubital tunnel syndrome, so music schools should teach students to be aware of these symptoms and take the proper preventative measures. Also, it appears that brass players may have the highest risk of developing carpal tunnel syndrome or cubital tunnel syndrome, so these musicians should be especially aware of early symptoms and take the necessary precautions to help prevent these neuropathies.

\section{References}

Atroshi, I., et al. (1999). Prevalence of carpal tunnel syndrome in a general population. Journal of the American Medical Association, 282(2): pp.153-158.

Bowie, E., K. Brimer, M. Kidder, et al. (2000). Median and ulnar nerve conduction studies in young adult violinists. Medical Problems of Performing Artists, 15:123-128.

Cummings, K., N. Maizlish, L. Rudolph, et al. (1989). Current trends: Occupational disease surveillance: Carpal Tunnel Syndrome. $M M W R, 38:$ 485-488.

Gerr, F. and R. Letz. (1998). The sensitivity and specificity of tests for carpal tunnel syndrome vary with the comparison subjects. I Hand Surg [Br]. 23B: 151-155.

Gohl, A., S. Clayton, K. Strickland, et al. (2006). Median and ulnar neuropathies in university pianists. Medical Problems of Performing Artists, 21: 17-24.

Kerwin, G., C.S. Williams, and J.G. Seiler III. (1996). The pathology of carpal tunnel syndrome. Hand Clin., 12: $243-245$.

Logue,E., S. Bluhm, M. Johnson, et al. (2005). Median and Ulnar Neuropathies in University Cellists. Medical Problems of Performing Artists, 20:70-76.

Robertson, C., B. Kin, and J. Saratsiotis. (2005). A review of compressive ulnar neuropathy at the elbow. Journal of Manipulative and Physiological Therapeutics, 28: 345.e1-345.e7

Strickland, James W. (2001). Carpal Tunnel Syndrome. NeuroMetrix. [Online] Available: http://www.neurometrix.com/papers_monos\%20pdf/StricklandMonograph.pdf

Table 1. Number of musicians who had values outside of the reference range, therefore confirming electrical evidence of carpal tunnel syndrome or cubital tunnel syndrome. 30 musicians were tested for DML-R, 29 for DML-L, 24 for F-wave Right, and 25 for F-wave Left.

\begin{tabular}{|l|l|l|l|l|}
\hline & DML-R & DML-L & F-wave Right & F-wave Left \\
\hline Brass & 1 & 2 & 3 & 2 \\
\hline Percussion & 0 & 0 & 0 & 0 \\
\hline String & 0 & 0 & 0 & 0 \\
\hline Woodwind & 0 & 0 & 0 & 0 \\
\hline
\end{tabular}

(overall, this represents one brass player with an abnormal DML-R, DML-L, and F-wave right, one brass player with an abnormal DML-L, F-wave right, and F-wave left, and one brass player with an abnormal F-wave right and F-wave left) 
Table 2. Results of chi-square tests comparing the incidence of carpal tunnel syndrome in the general population to the incidence in each musician population. Data represent 8 brass musicians, 8 percussion musicians, 5 string musicians, and 9 woodwind musicians.

\begin{tabular}{|c|c|}
\hline Musician Group & P-value \\
\hline Brass & $3.94 \mathrm{E}-5$ \\
\hline Percussion & 0.5271 \\
\hline String & 0.6171 \\
\hline Woodwind & 0.5145 \\
\hline
\end{tabular}

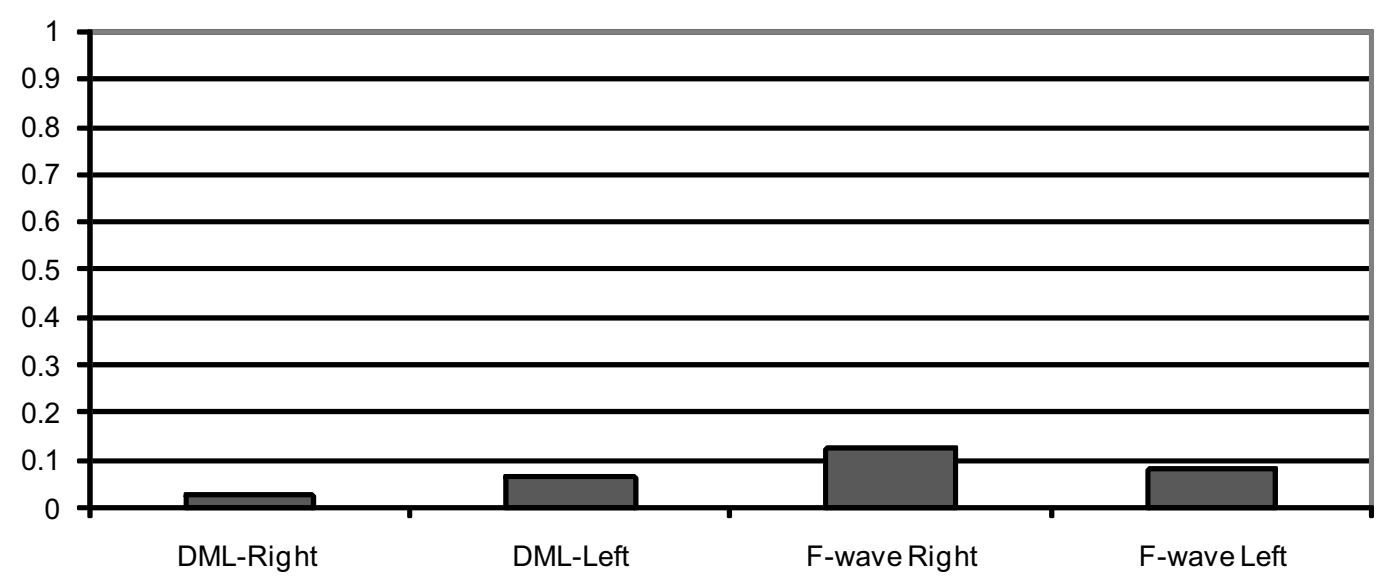

Figure 1. Proportion of all musicians who had values outside of the reference range. Data describe 30 musicians for DML-R, 29 musicians for DML-L, 24 musicians for F-wave right, and 25mu sicians for F-wave left.

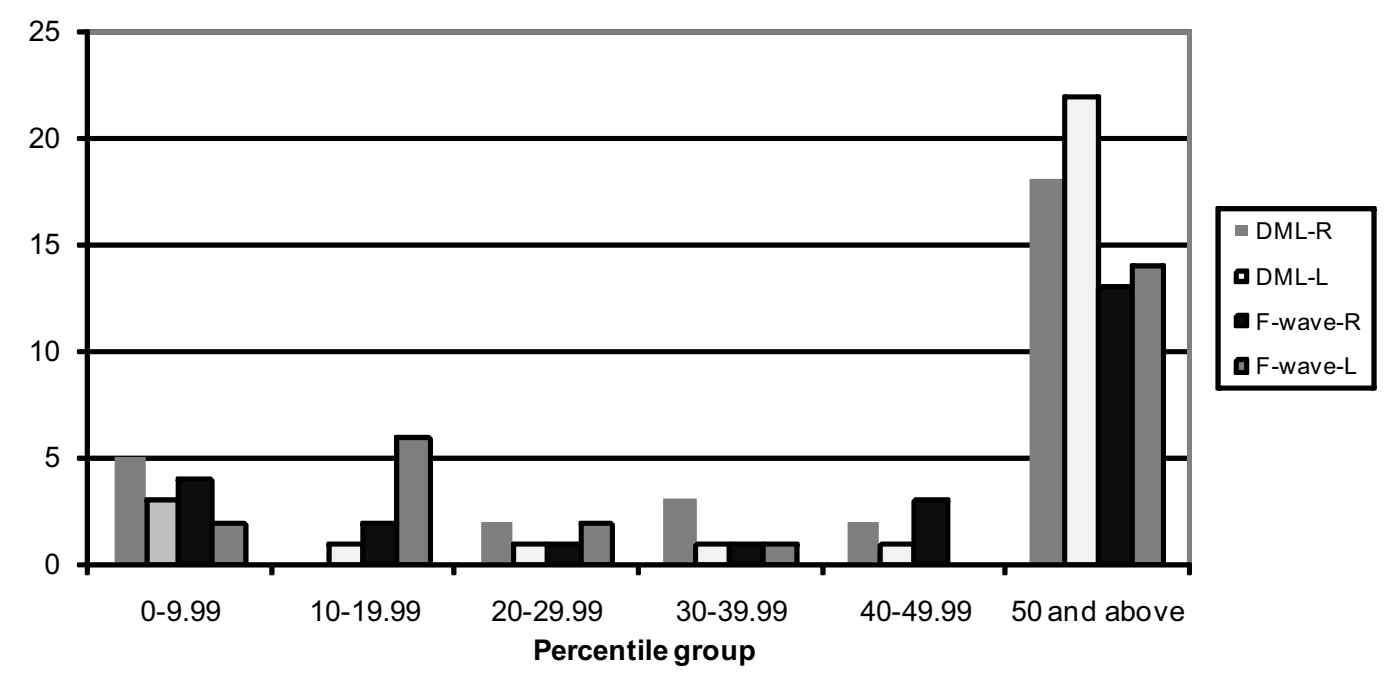

Figure 2. Distribution of percent tiles for all musicians. Data represent 30 musicians for DML-R, 29 for DML-L, 24 for F-wave right, and 25 for F-wave left. Higher percentiles represent faster conduction and therefore healthier nerves. 


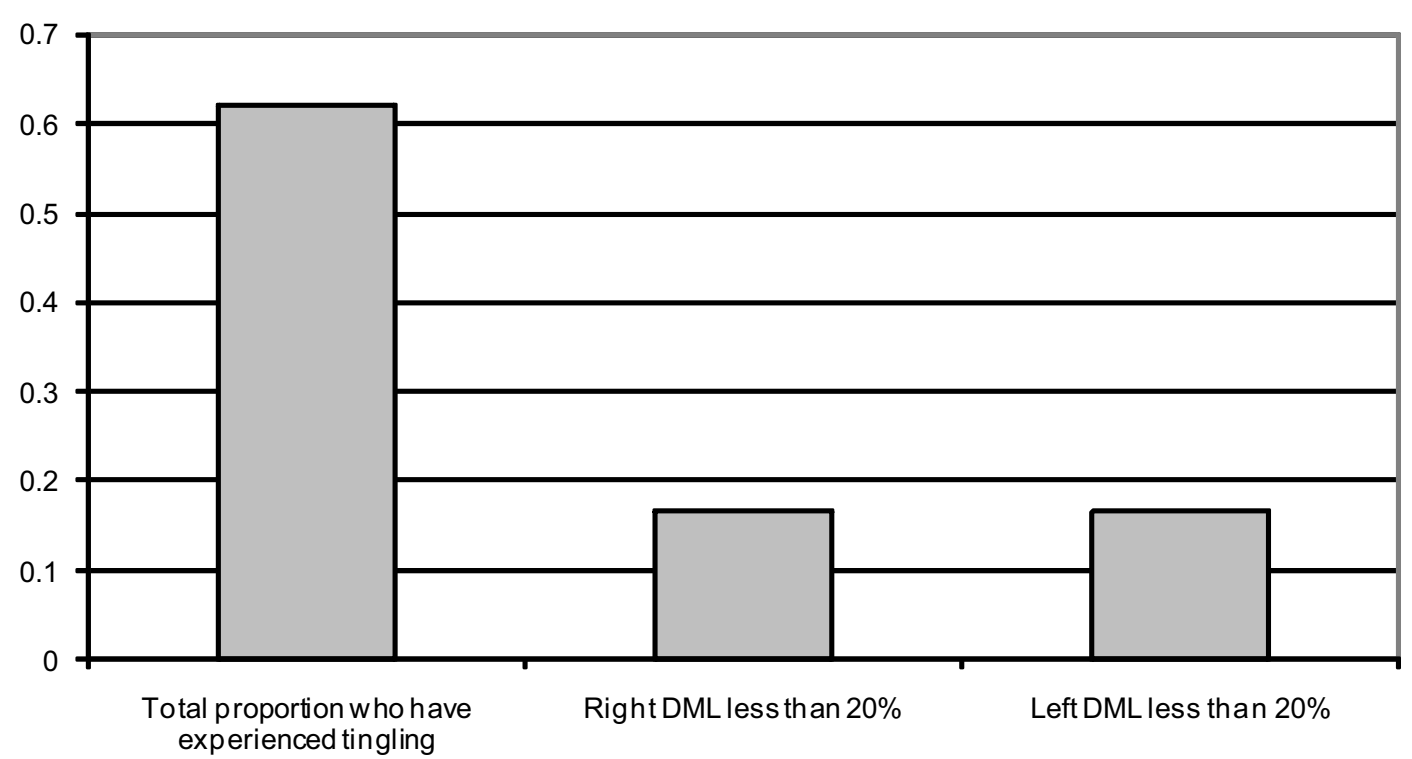

Figure 3. Proportion of musician who have experienced tingling, and of those musicians, the proportion that actually had electrophysiologic values below the $20^{\text {th }}$ percentile. Data represent 29 total musicians.

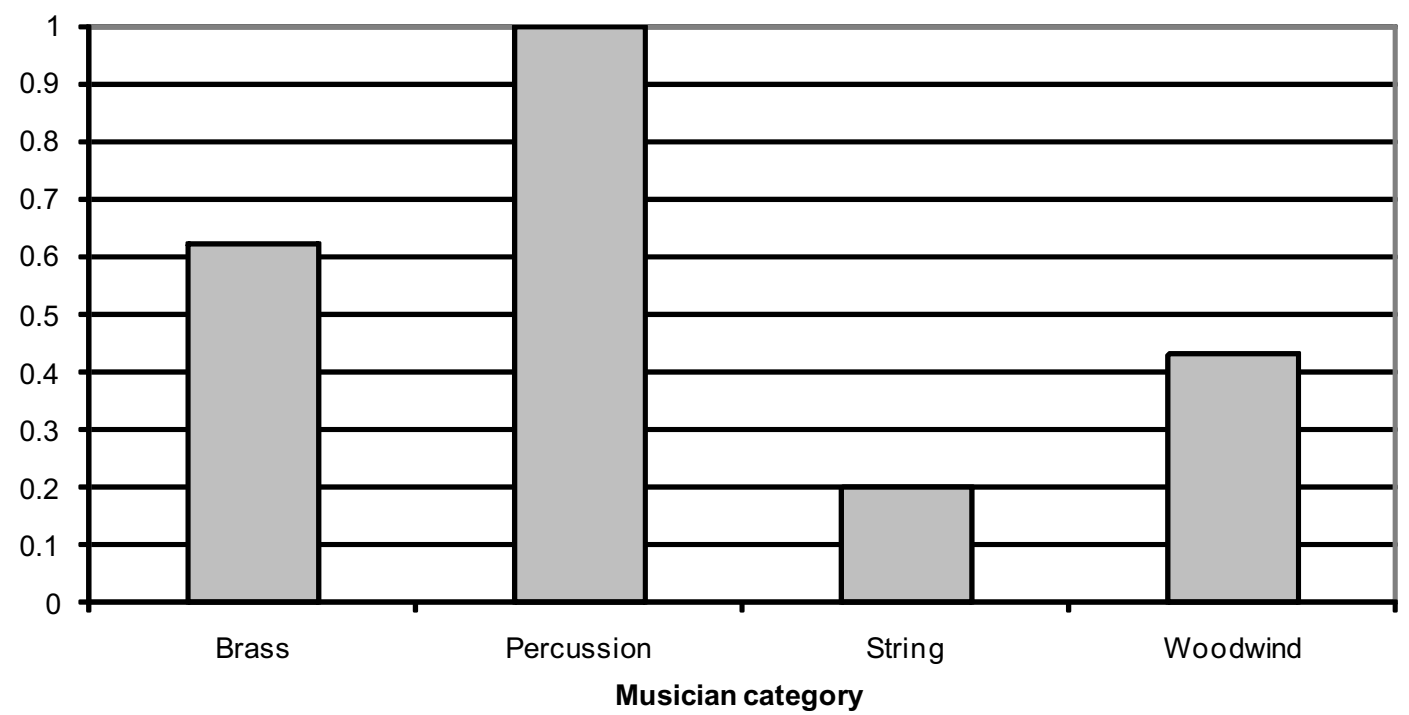

Figure 4. Proportion of musicians who have had reports of tingling in their fingers. Data represent 8 brass musicians, 8 percussion musicians, 5 string musicians, and 8 woodwindmusicians. 


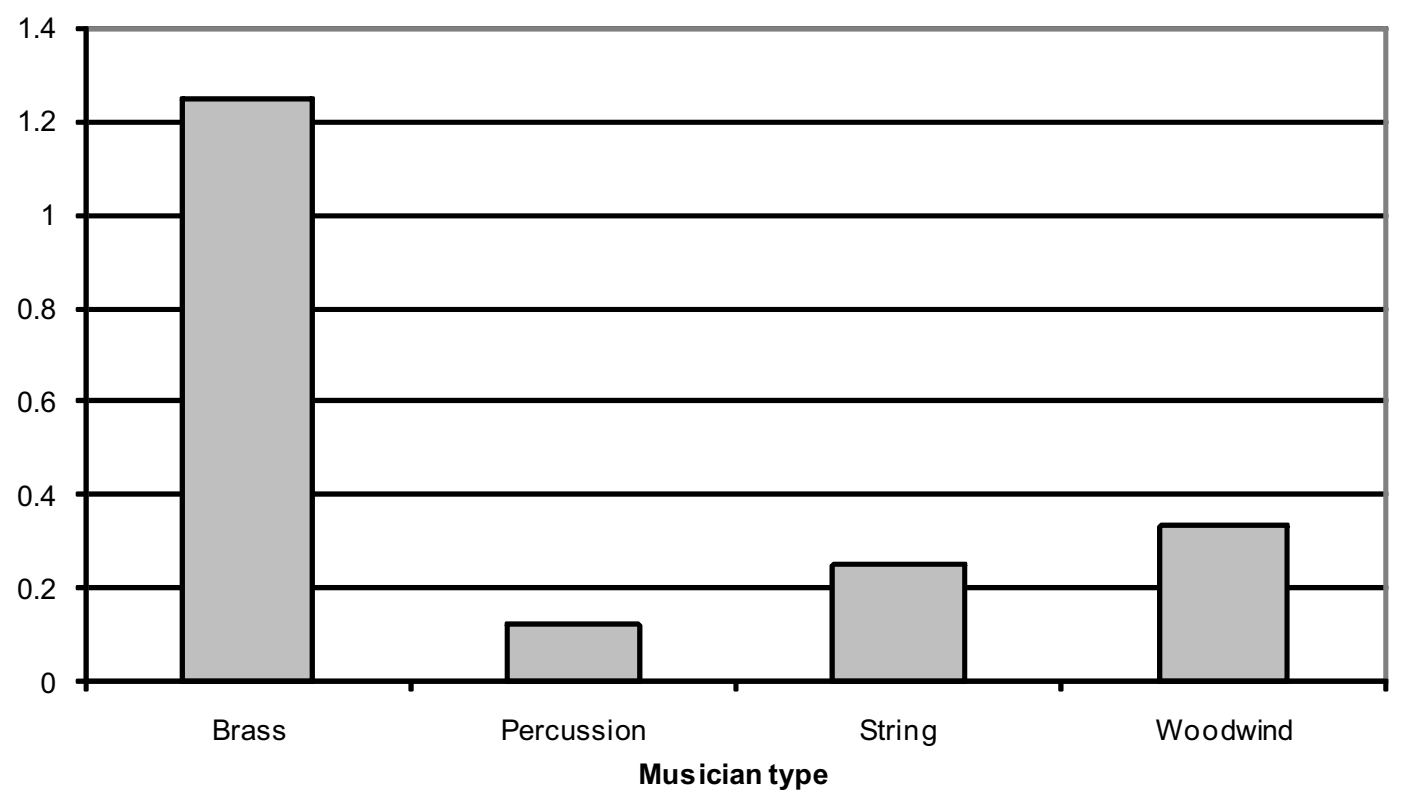

Figure 5. Average number of median nerve flags per musician. Data represent 8 brass musicians, 8 percussion musicians, 5 string musicians, and 9 woodwind musicians.

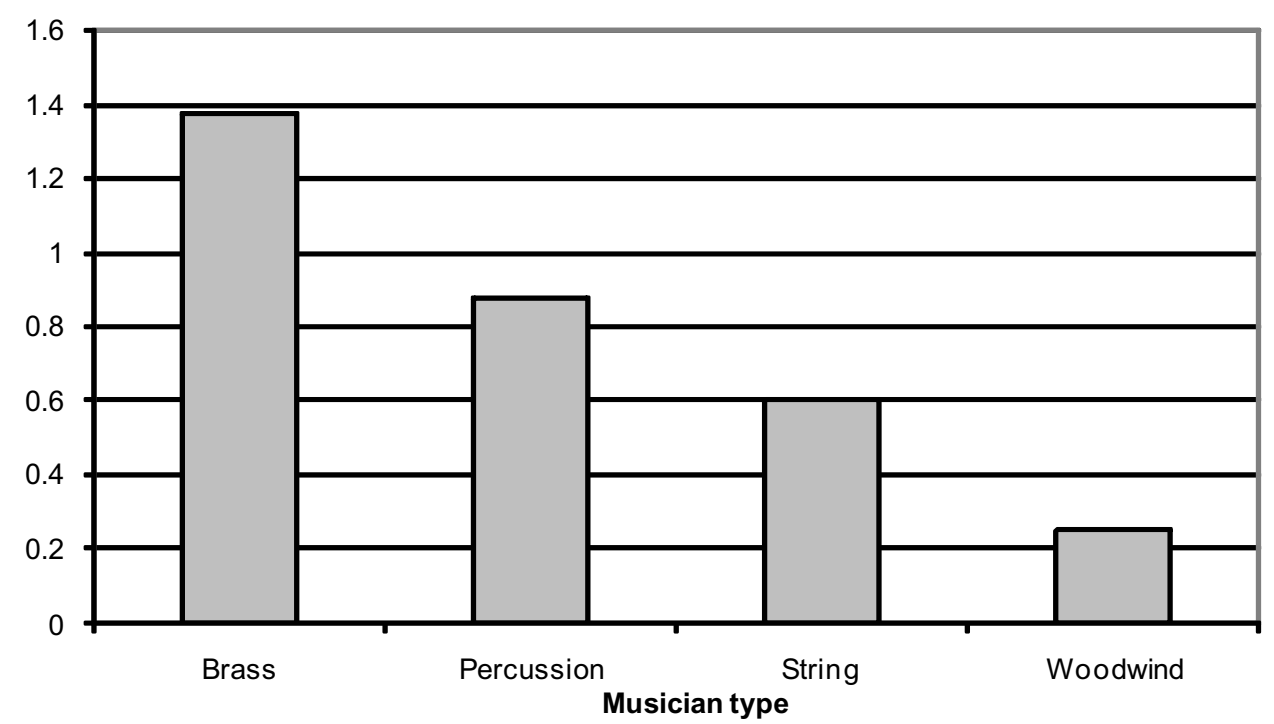

Figure 6. Average number of ulnar flags per musician. Data represent 7 brass musicians, 8 percussion musicians, 5 string musicians, and 8 woodwind musicians. 


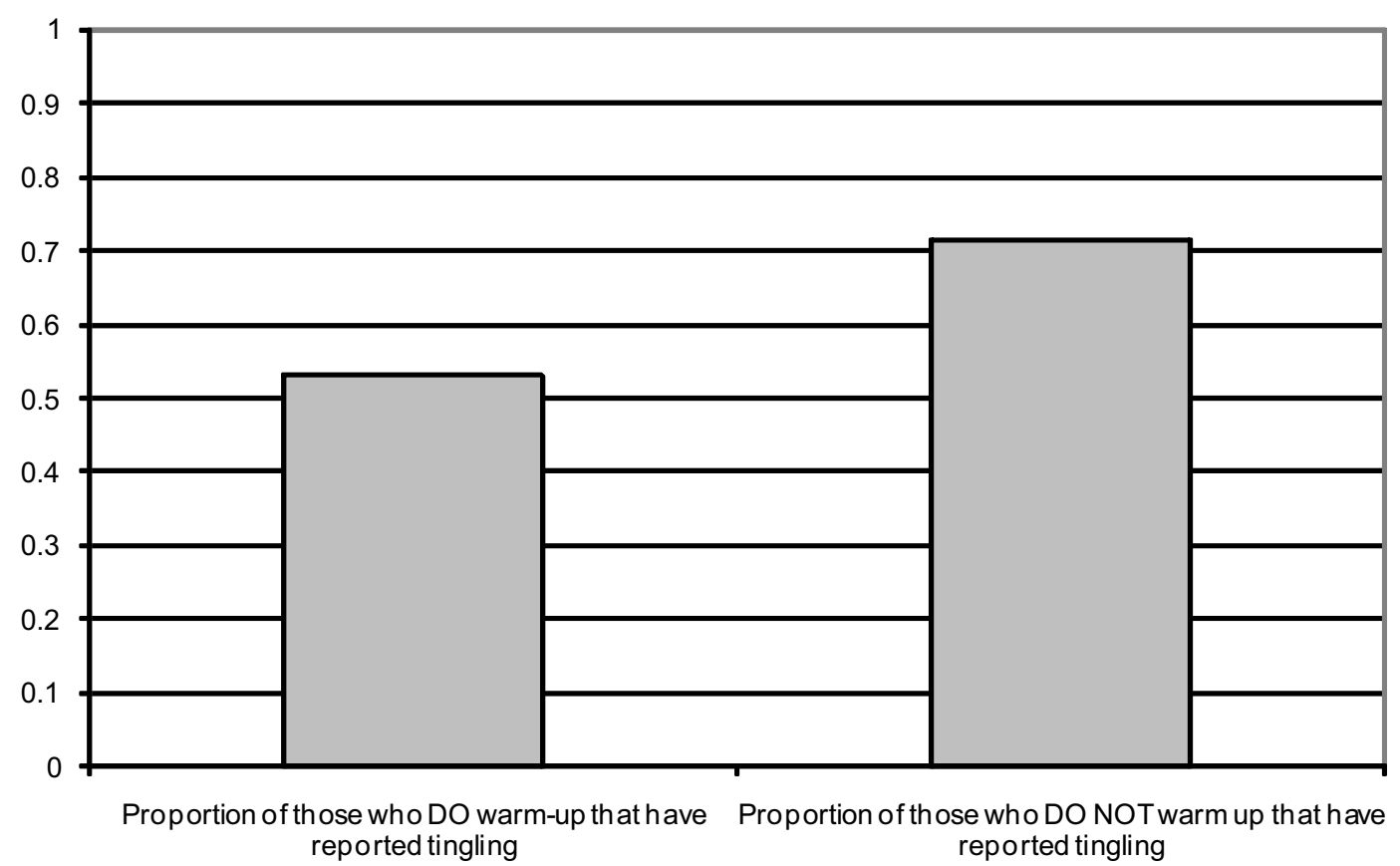

Figure 7. The correlation between performing a warm-up routine and reported experiences of tingling. Data represent survey results of 29 musicians.

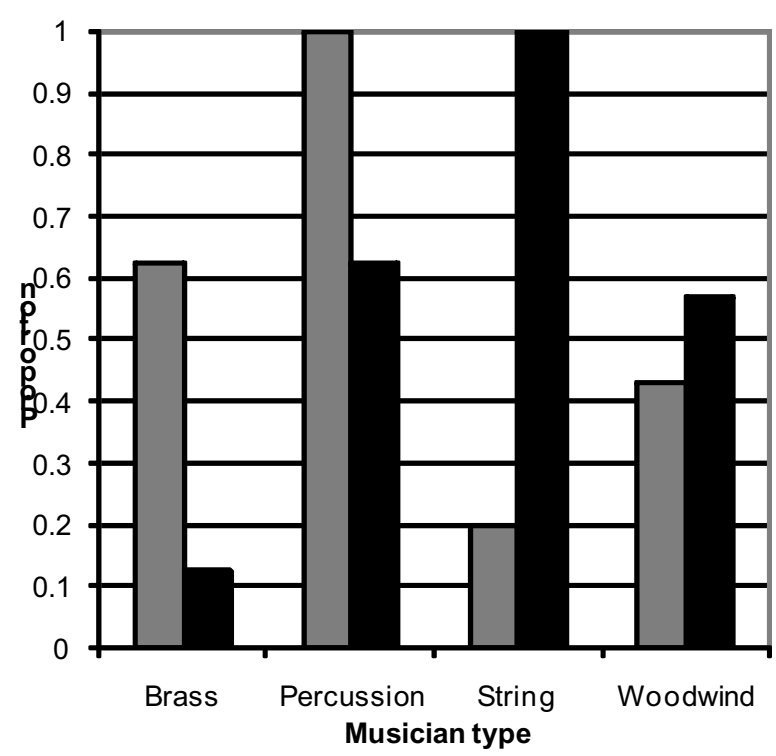

DProportion who have
experienced tingling
Proportion who warm-up prior to
playing

Figure 8. The correlation between tingling and warm-up for each musician group. Data represent the survey results for 8 brass musicians, 8 percussion musicians, 5 string musicians, and 8 woodwind musicians. 